\title{
Lack of Fpr2/Fpr3 receptors alters the structure and function of pituitary corticotrophs
}

Maria Mahmood, Helen Christian

\section{ABSTRACT}

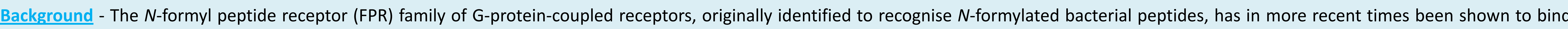

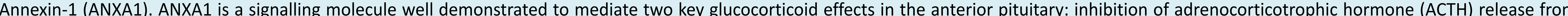

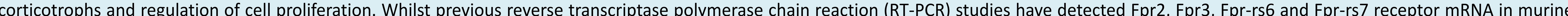

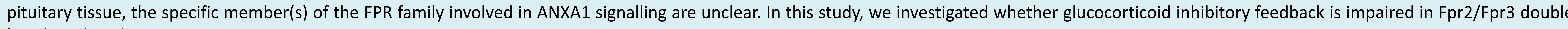
knockout (DKO) mice.

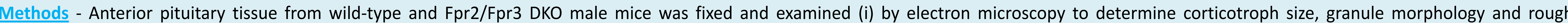

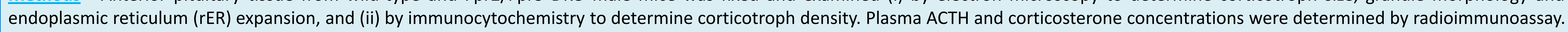

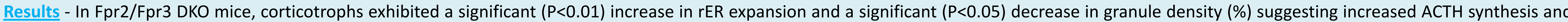
secretion corresponding with elevated levels of plasma ACTH $(P<0.05)$ and corticosterone $(P<0.05)$. Fpr2/Fpr3 DKO mice exhibited a significant $(P<0.01)$ increase in corticotroph cell density

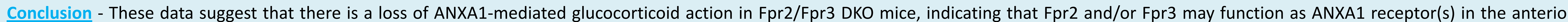
pituitary.

\section{Introduction}

Corticotrophs are endocrine cells of the anterior pituitary at the centre of HPA axis regulation, responsible for adrenocorticotrophic hormone (ACTH) cretion under the positive control of hypothalamic corticotropin-releasin hormone (CRH) and the inhibitory control of plasma glucocorticoids (GCs). A substantial body of evidence supports the role of Annexin 1 (ANXA1) as paracrine mediator of glucocorticoid (GC) feedback in the anterior pituitary. experimental observations are consistent with the proposal that GC-induced ANXA1 externalisation from folliculostellate cells is associated with inhibition of ACTH secretion from adjacent corticotrophs.

The antiproliferative effects of glucocorticoids are thought to be at least partially mediated by ANXA1. Indeed, in ANXA1 knockout male mice a fourfold increase in the number of corticotrophs ${ }^{2}$ has been noted with no associated change in hypothalamic CRH expression, suggesting that ANXA1 directly influences corticotroph proliferation in the anterior pituitary.

The FPR family of G-protein-coupled receptors consists of three human genes designated FPR1, FPR2, and FPRL2 on chromosome 19 in conserved synteny with the mouse FPR gene cluster on chromosome 17. Genomic screening in mice using CDNA probes for human FPRs has revealed eight distinct mouse genes: Fpr1, Fpr2, Fpr3, Fpr-rs3, Fpr-rs4, Fpr-rs5, Fpr-rs6 and Fpr-rs7. ${ }^{3}$ Murine Fpr2 and Fpr3 are highly homologous (82\%) receptors transcribed from the same DNA template strand and share structural and pharmacological properties with the human FPR2 receptor. ${ }^{4}$

The identity of the precise FPR subtype(s) involved in glucocorticoid feedback in the anterior pituitary is currently unclear.

Hypothesis: ANXA1-mediated glucocorticoid feedback in the anterior pituitary is impaired in Fpr2/Fpr3 double knockout (DKO) male mice.

\section{Methods}

Animals

作 cells as first described by Dufton et al..$^{5}$ All wild-type and Fpr2/Fpr3 DKO mice were sacrificed at 3 months of age. Trunk blood was collected for assay of plasma ACTH and corticosterone.

Detection of Fpr3 by Western blot analysis

Fpr3 was detected by overnight incubation of proteins extracted from pituitary tissue by sonication with a rabbit antiFpr3 polyclonal antibody 1:1000 dilution (Abcam, Cambridge, UK) then anti-rabbit IgG conjugated to horseradish peroxidase 1:5000 dilution (Sigma Chemical Co., Poole, UK).

Quantitative Electron Microscopy:

In order to identify the corticotrophs in the pituitary immunogold labelling for ACTH was used. The sections were viewed with a JEOL 1010 transmission electron microscope (JEOL, Peabody, MA, USA)

Six micrographs of corticotrophs per animal were taken at a magnification of $x 4000$ for analysis of cell size, granule morphology and rough endoplasmic reticulum (rER) expansion. Negatives were scanned into Adobe Photoshop (version 5.5) and transferred to Axiovision (version 4.5) software for image analysis. The total granule area and granule density (\%) calculations were carried out using Microsoft Office Excel 2007 (version 12.0). Expansion of rER was assessed visually from electron micrographs and quantified on a scale of 0-3, 0 being no expansion and 3 the Wast expansion. In all cases, the analyst was blind to the sample code.

Immunofluorescence

Sections of anterior pituitary tissue were examined using a TCS confocal microscope (Leica Corp, Microsystems, Wetzlar, GmbH, Germany). Corticotroph density for wild-type and Fpr2/Fpr3 DKO mice was quantified by counting ACTH immunopositive cells per $100 \mu \mathrm{m} \times 100 \mu \mathrm{m}$ of section (determined by Axiovision 4.2) for eight different sections. Plasma ACTH and corticosterone measurement

Plasma ACTH and corticc

Statistical analysis

Quantitative electron microscopy data (expressed as mean \pm standard error of the mean SEM, $n=4$ ) were shown to be non-normally distributed (Shapiro-Wilks test). Subsequent analysis was undertaken using non-parametric methods: Kruskal-Wallis test with post hoc analysis performed using the Mann-Witney U Test. For ACTH and corticosterone radioimmunoassay data (expressed as mean $\pm \mathrm{SEM}, \mathrm{n}=8$ for both) and corticotroph density data (expressed as mean \pm SEM, $n=8$ ) preliminary analysis indicated the data were normally distributed (Shapiro-Wilks test). Parametric methods were therefore used for analysis: one-way ANOVA with post hoc analysis performed using the Bonferroni test. In all cases, differences were considered significant if $\mathrm{P}<0.05$.

\section{Results}

Figure 1:

Fpr3 expression in the

Fpr3 expression in the

WT mice

Figure 2:

ANXA1 expression in

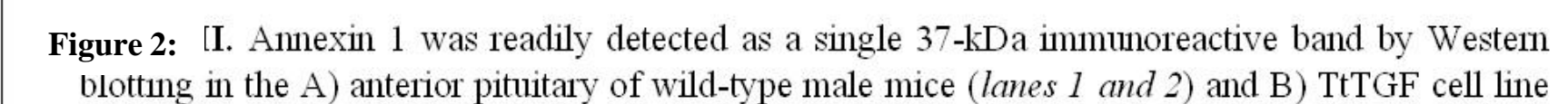

of WT mice

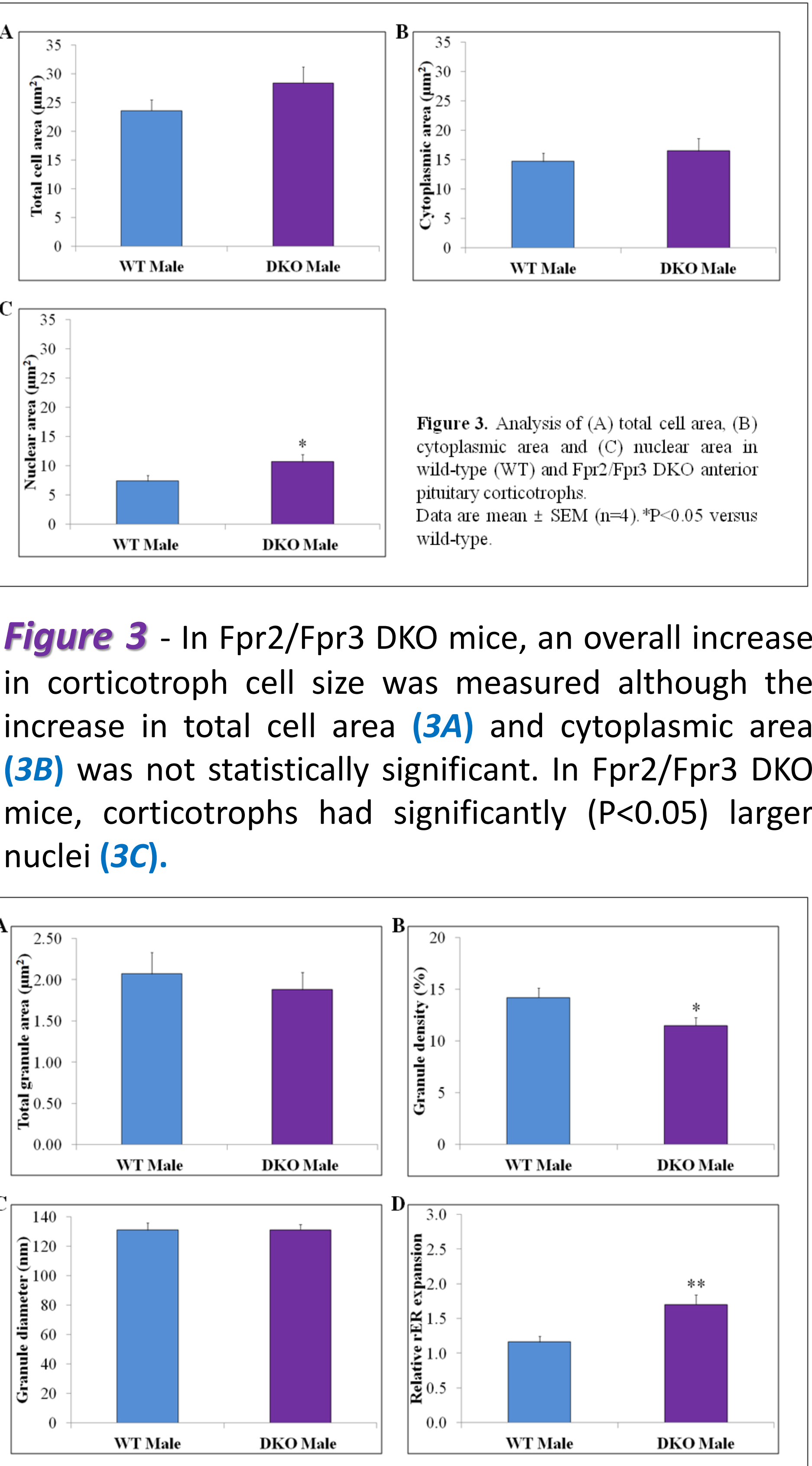

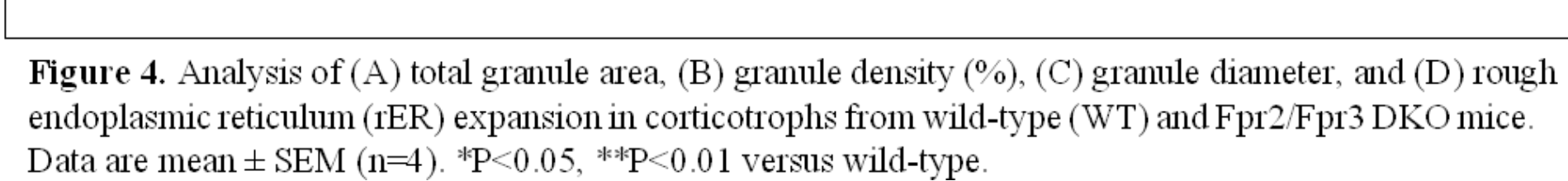

Figure 4: In Fpr2/Fpr3 DKO mice, corticotrophs had reduced total granule area although this change was not significantly different to wild-type corticotroph $(4 A)$. Granule density $(\%)$ was significantly $(P<0.05)$ reduced in Fpr2/Fpr3 DKO mice compared with wildtype (4B). In Fpr2/Fpr3 DKO corticotrophs, granule diameter was not affected (4C) however the roug endoplasmic reticulum ( $r E R)$ was significantly $(P<0.01)$ expanded (4D)

\section{Discussion}

In the present study, we have demonstrated that Fpr2 and/or Fpr3 deficiency is associated with increased ACTH synthesis and secretion and a dramatic increase in corticotroph density consistent with the hypothesis that glucocorticoid (GC) inhibitory feedback is impaired in Fpr2/Fpr3 double knockout (DKO) male mice.

The reduction in total granule area and granule density (\%) observed in Fpr2/Fpr3 DKO corticotrophs suggests that in the absence of GC inhibition, ACTH granules are released causing depletion of the intracellular stores. The diameter of corticotroph granules is unaffected in the absence of ANXA1 (in ANXA1 knockout mice) ${ }^{2}$ or its putative receptor(s) Fpr2 and/or Fpr3 (in Fpr2/Fpr3 DKO mice). This finding is supported by previous studies demonstrating that ANXA1 signalling via Fpr2 and/or Fpr3 may prevent granule exocytosis through formation of an actin barrier at the plasma membrane ${ }^{6}$ In this way, GC inhibition of ACTH release does not modify ACTH granules, thus granule diameter is unchanged when ANXA1 or Fpr2/Fpr3 function is disrupted.

The increase in corticotroph density in Fpr2/Fpr3 DKO mice suggests that ANXA1 binds Fpr2 and/or Fpr3 to mediate the antiproliferative actions of GCs in the anterior pituitary. In Fpr2/Fpr3 DKO mice, the four-fold increase in corticotroph density compared with wild-type mice may additionally reflect the function of ANXA1 in pro-apoptotic signalling.

In this study, Western blot analysis also indicated that Fpr3 is expressed in the hypothalamus. Therefore, an alternative explanation for corticotroph population expansion in Fpr2/Fpr3 DKO mice may be loss of ANXA1-mediated GC action at the hypothalamus, including $\mathrm{GC}$ suppression of $\mathrm{CRH}$ mRNA to regulate the mitogenic effects of CRH on the anterior pituitary. ${ }^{8}$ Hence, the aim of future studies will be to dissect the relative contribution of GC feedback on the hypothalamus to changes in corticotroph structure and function observed in Fpr2/Fpr3 DKO mice.

Additionally, in this study we have demonstrated Fpr3 expression in the folliculostellate (FS) TtT/GF cell line, which to our knowledge has not been shown previously. This may represent an autocrine feedback loop whereby externalised ANXA1 binds Fpr3 on FS cells to regulate its own release.

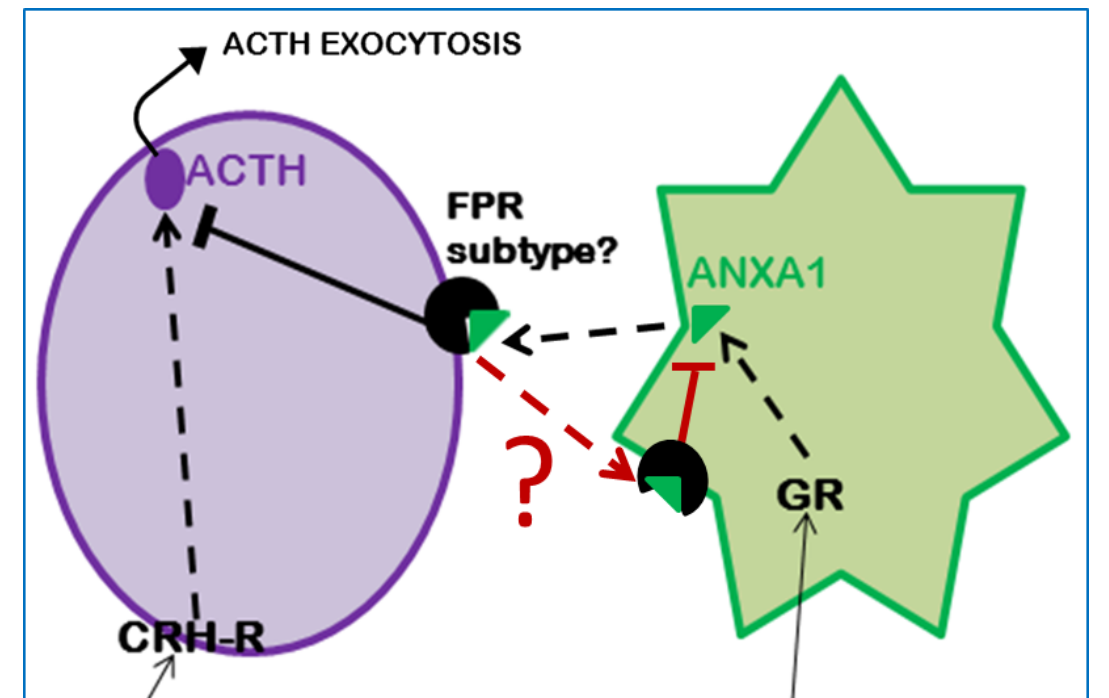

CRH

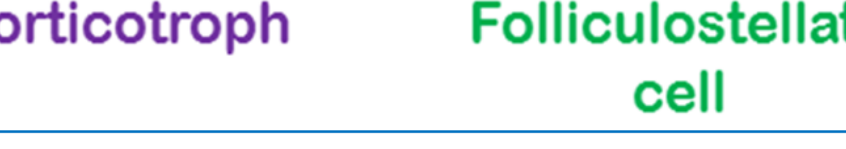

